\title{
La centralidad del trabajo y su relación con variables macroeconómicas. Una lectura del caso mexicano en comparativa
}

\author{
The centrality of work and its relationship with macroeconomic variables. \\ A reading of the mexican case in comparative
}

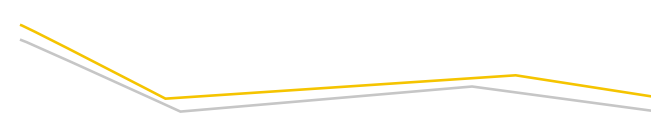

Carlos Mejía Reyes*®

Mejía Reyes, C. (2017). La centralidad del trabajo y su relación con variables macroeconómicas. Una lectura del caso mexicano en comparativa. Investigación y Ciencia de la Universidad Autónoma de Aguascalientes, 25(71), 68-76.

RESUMEN

El presente estudio analizó la importancia del trabajo frente a otras esferas de la vida en una muestra de informantes mexicanos, con datos extraídos de la última Encuesta Mundial de Valores (World Values Survey Association, en inglés) durante el periodo 20102014 y sus fluctuaciones desde 1990. La finalidad del análisis es explicar los comportamientos estadísticos de la centralidad del trabajo y su relación con variables macroestructurales. La hipótesis de partida es que la importancia del trabajo en México es elevada en comparación con otras esferas vitales. La razón de ello se manifiesta por las condiciones materiales y de bienestar social ínfimas, tal como lo predice la teoría del posmaterialismo de Ronald Inglehart.

\section{ABSTRACI}

This study analyzed the centrality and importance of work against other spheres of life in a sample of

Palabras clave: centralidad del trabajo; Encuesta Mundial de Valores; posmaterialidad; México.

Keywords: work centrality; World Values Survey; postmaterialism; Mexico.

\section{Recibido: 18 de julio de 2016, aceptado: 10 de febrero 2017}

* Área Académica de Sociología y Demografía, Instituto de Ciencias Sociales y Humanidades, Universidad Autónoma del Estado de Hidalgo. Carretera Pachuca-Actopan km. 4.5, Colonia Campo de Tiro, C. P. 42039, Pachuca de Soto, Hidalgo, México. Correo electrónico: mejiareyescarlos@gmail.com

Trabajo derivado de la investigación doctoral "La centralidad del trabajo. Un análisis comparativo de España y Alemania", tutorada por el Dr. Antonio Martín Artiles de la Facultat de Ciències Polítiques i de Sociología de la Universitat Autònoma de Barcelona, España.

$凶$ Autor para correspondencia mexican informants extracted from the latest World Values Survey (World Values Survey Association) from 2010 to 2014 lapse and its fluctuations since 1990. The purpose of the analysis was to explain the statistical behavior of the centrality of work and its relationship with structural macro variables. The hypothesis is that the importance of work in Mexico is high compared to other vital areas. The reason for this is manifested by the negligible material conditions and social welfare, as predicted by Ronald Inglehart's theory of postmaterialism.

\section{INTRODUCCIÓN}

Para comprender la centralidad del trabajo, el mismo se define básicamente como la actividad ejecutada con relativo esmero y disciplina destinada a la producción de valores de uso por el agente que lo elabora y el colectivo al cual pertenece, cuya finalidad es la recompensa material o social; es decir, una actividad instrumental (Noguera, 2000, 2002). Es comprendido también como una capacidad humana que transforma la naturaleza y posteriormente genera posibilidades de concebir la acumulación originaria de capital, el despliegue de la industria, mercado y la circulación de mercancías a diferentes escalas (Köhler, \& Martín Artiles, 2010; Rieznik, 2007).

Sin embargo, el interés conceptual de los autores de esta investigación acerca del trabajo obedece a otro tipo de utilidad. Es decir, consideran al trabajo en su sentido social, cultural y normativo que representa beneficios no solo materiales, sino colectivos, éticos y políticos diversos (Noguera, 2002). Es decir, que el trabajo es un concepto semántico, no ontológico, cuya consideración depende de cada 


\section{InVESTIGACIÓn Y CIERCIA DE LA UחIVERSIDAD AUTÓnOMA DE RGUASCALIERTES}

cultura o época, que se relaciona con significados de prestigio, jerarquía, estigmas colectivos, etcétera (Sahlins, 1997).

La centralidad del trabajo es una temática ampliamente estudiada en las ciencias sociales. Se pueden reseñar algunos aportes iniciales, como los postulados teórico-económicos de Smith (2010) quien ubicó al trabajo como la fuente de la riqueza material de las naciones y de la cohesión social. Marx (1984), quien retoma críticamente la anterior tesis y aporta la distinción entre trabajo abstracto/ concreto en el desarrollo capitalista inicial consideró que el modo de producción es la base de las contradicciones sociales que producen la historicidad. Por tanto, la relación social del trabajo figura como elemento central de análisis. Weber (1999), a partir del estudio del protestantismo como ética religiosa y de vida, refiere su influencia en la constitución del capitalismo europeo occidental, donde la significación cultural del trabajo toma relevancia como espíritu del sistema económico.

Dichos tratamientos clásicos son teorizaciones de lo que las sociedades modernas e industriales burguesas cimentaron como la ideología del trabajo, según la cual fundamenta la organización colectiva a nivel no solamente económico, sino social, político y cultural (Arendt, 2014; Bauman, 2004; Habermas, $1992,1999,2002)$. Esta es la acepción que nos interesa desarrollar; es decir, la importancia del trabajo y su significado en contextos particulares, así como en función de procesos sociales contemporáneos.

Como ejemplo de las distintas acepciones del trabajo, en las sociedades preeconómicas tuvo importancia por su carácter social al entenderse como el vehículo para el prestigio social regido por máximas y calendarios religiosos (Méda, 1998; Molina, \& Valenzuela, 2007; Veblen, 2005). En la Grecia clásica representaba tareas absolutamente degradantes y de poco aprecio (Arendt, 2014). Lo mismo para el imperio romano (Méda, 1998); sin embargo, en la Edad Media se reinterpretó el concepto hasta alcanzar una máxima que sanciona la inactividad infructuosa para la contemplación a su Dios y la vida material; abriendo paso a la idea de salvación a partir de la laboriosidad (Sanchis Gómez, 2004).

Con la reforma protestante la nueva ojeada de las "santas escrituras" no sólo suponía la inexistencia de paraísos extraterrenales, sino que ahora la existencia misma se convertía en la actividad para alabar a su deidad mediante la labor encomendada: trabajar (Weber, 1999). En las sociedades industriales capitalistas, entendida la actividad como trabajo asalariado, se subvirtieron las formas precapitalistas del trabajo ciñéndolas al cálculo de la ganancia mediante coacciones operativas, lo que provocó giros súbitos de valores, modos de vida, de referirse $u$ orientarse en la acción. En suma, de hacer del trabajo la vida misma, o sea la alienación (Gorz, 1995; Méda, 1998); es decir, que se ha considerado como una categoría medular en la vida de los sujetos modernos (Antunes, 2005).

El papel del trabajo en la subjetividad de los individuos conforma el contenido que alimenta la directriz, valoración y expectativas de actividad en los procesos sociales ante situaciones concretas, particularmente referidas a la actividad de trabajar y sus derivaciones. Por tanto, la centralidad del trabajo se entiendo como el conjunto de creencias, definiciones y el valor que individuos y grupos atribuyen al trabajo (MOW International Research Team, 1987; Noguera, 2002). Se comprende entonces al trabajo como central, ya que alude al grado de importancia que esta actividad posee en la vida de los sujetos. Esta es comprendida de forma absoluta, es decir a la importancia general otorgada al trabajo en general por parte de los individuos, y de forma relativa, entendida como la importancia del trabajo con respecto a otras esferas de la vida en la situación concreta de encontrarse en un empleo (García Martínez, \& Berrios Martos, 1999; Kanungo, 1983; MOW International Research Team, 1987).

Investigaciones empíricas contemporáneas, que desde el matiz cuantitativo se han convertido en referencias obligadas de estudios de la centralidad del trabajo son, por ejemplo, el grupo conocido como "El significado del trabajo", en inglés The Meaning of Working (MOW International Research Team), cuya investigación internacional a nivel comparativo tuvo como meta revisar el compromiso en la labor a partir del significado que los actores le otorgan a la actividad mediante una encuesta de 15, 000 informantes de nueve países (MOW International Research Team, 1987). Derivado de este grupo, Snir y Harpaz $(2002,2005)$ han realizado investigaciones que analizan la prioridad de esferas de la vida, en las que el trabajo conforma una de ellas. También se investigó la importancia que tendría el trabajo si los informantes ganaran la lotería o heredaran sumas millonarias (Snir, 2011). 
IIVESTIGACIÓn Y CIERCIA DE LA UNIVERSIDAD AUTÓNOMA

70
Por otra parte, Schwartz (1999), quien a partir de estudios sobre valores humanos como principios guía analiza el trabajo en facetas valorativas como la centralidad, normas y metas en el empleo, y de forma comparativa genera una tipología de países con alta centralidad al trabajo. Inglehart (1971, 1991, 1998) ha estudiado al trabajo como valor y su relación con condiciones económicas desahogadas. Méda y Davoine (2008) contrastan la importancia del trabajo entre Francia y Europa mediante la misma encuesta que el presente estudio. En el caso de España, Veira Veira y Romay Martínez (1998), con la misma fuente de datos, lo estudian tanto en contexto nacional como regional. Estos antecedentes incentivan a realizar una exploración del tema para el caso de México utilizando la última encuesta 2010-2014 con el objetivo de analizar la centralidad del trabajo frente a otras esferas de la vida y su relación con variables de bienestar social y material.

\section{MATERIALES Y METODOS}

Esta investigación se propone haceruso de encuestas de valores, particularmente la World Values Survey (WVS, s. f.) o Encuesta Mundial de Valores del ciclo 2010-2014. La particularidad de esta encuesta como fuente base del estudio radica en la naturaleza de los objetivos con la que fue creada: entender los cambios en percepciones, creencias, motivaciones y valores de las personas en el mundo por medio de encuestas representativas. La técnica de recogida de datos se efectúa en trabajo de campo cara a cara con el o la entrevistada, los tipos de muestra son nacionales y estratificada en varias etapas, el lenguaje utilizado es el propio de cada país y el procedimiento de ponderación es por sexo y edad. Todas las encuestas fueron realizadas a informantes mayores de edad.

El criterio de selección de la variable dependiente de la encuesta está en función de los ejemplos que otros estudios aportan para analizar el mismo fenómeno con la misma fuente de datos (Davoine, \& Méda, 2008; Inglehart, 1991, 1998; Méda, \& Vendramin, 2013; Veira Veira, \& Muñoz Goy, 2004; Veira Veira, \& Romay Martínez, 1998), así como de la sistematicidad con que fue reunida la información al respecto en las encuestas previas. Así, la variable a utilizar es de tipo Likert que versa de la siguiente manera: "Dígame, por favor, qué grado de importancia tiene en su vida uno de los siguientes aspectos: El trabajo". Y las dimensiones son: 1) Muy importante, 2) Algo importante, 3) No muy importante, y 4) Nada importante.

La muestra a utilizar consta de $\mathrm{N}=2000$ casos; 999 hombres (50\%) y 1, 001 mujeres (50\%). El porcentaje de respuesta a la variable considerada es de $100 \%$. El primer ejercicio analítico descriptivo de la variable dependiente es la revisión de la centralidad del trabajo ante otras esferas de la vida. La forma de proceder para ello tiene como antecedente los ejercicios realizados por Kanungo (1982) y MOW International Research Team (1987), que posibilita su revisión en estos términos. El procedimiento de análisis que se realiza es la comparación de la importancia que posee la labor frente a otras esferas (ocio, familia, religión, política, amigos) a partir del comparativo de la variable dependiente con otras construidas bajo la misma estructura. La interpretación de los datos se realiza a partir de los resultados cuando el valor se acerca o aleja al de la categoría de referencia; en este caso: Muy importante.

Otra de las formas en que los autores del presente analizan la centralidad del trabajo pero ahora en su categorización absoluta es mediante la relación con variables macroeconómicas para verificar la influencia de las condiciones materiales en los valores sociales y particularmente en la centralidad del trabajo. La correlación es la técnica utilizada, ya que como cálculo estadístico habilita la posibilidad de dar cuenta del comportamiento de la variable dependiente con otras de externas a la base de datos para así explicar su relación (Guijarro Martínez, 2013). Con la cuantificación de su nexo puede sugerirse una correspondencia de causalidad mutua, ya que únicamente se realiza entre dos variables independientes entre sí (Arriaza Balmón, 2006).

Las variables comúnmente utilizadas para la explicación de las relaciones entre cambios valorativos y condiciones materiales han utilizado el Producto Interno Bruto per cápita, índices de crecimiento económico (Inglehart, 1998). Sin embargo, para otros desarrollos de la propuesta no solo las variables macroeconómicas son las adecuadas o en su caso únicas para los estudios, sino que se han propuesto también índices llamados macrosociales, como de desarrollo humano y de desigualdad GINI (Ros García, 2002). Para ello, los autores del presente sintetizan los datos absolutos en lapsos promedio de 4 años con fines de hacer coincidir los datos con los tiempos de levantamiento de cada encuesta. 


\section{IIVESTIGAGIÓn Y CUERCIA DE LA UחIVERSIDAD AUTÓNOMA DE RGUASCALIERTES}

\section{RESULTADOS}

La importancia del trabajo en México a través de 20 años muestra un comportamiento estadístico peculiar (figura 1). Como se aprecia, hay dos etapas de alta centralidad del trabajo. La primera en las muestras de 1990-1994 y 1995-1998, en las que la valoración como "Muy importante" fue de 66.9 y 61 puntos porcentuales, respectivamente; cuya orientación es a la baja en porcentaje de encuestados con alta valoración al trabajo. Inmediatamente se percibe un crecimiento abrupto hasta alcanzar 86.1, 84.9 y 87.0.

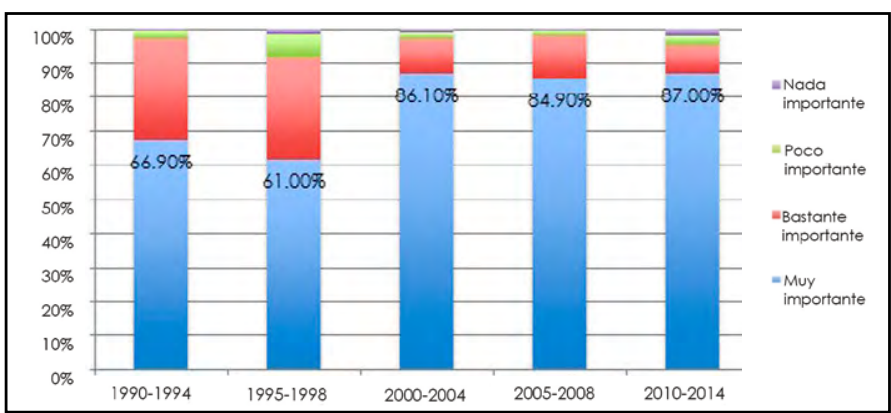

Figura 1. Importancia del trabajo en la vida en México desde 1990 hasta 2014 en porcentaje.

Elaboración propia con datos de WVS (s. f.).

Por tanto, la tendencia es hacia un aumento a considerar al trabajo como valioso; esto de manera acentuada desde inicio del milenio hasta la reciente encuesta. En la última encuesta, y a nivel comparativo con países americanos encuestados, se puede analizar la peculiar conducta de los datos contrastando la centralidad del trabajo con otras esferas de la vida (Centralidad del trabajo relativa) (tabla 1). El promedio de América posiciona al trabajo como la segunda prioridad (3.57) por debajo de la familia (3.98); mientras que Europa lo valora en menor medida (3.32) que el promedio mundial (3.47). Consecuentemente, América valora más al trabajo que Europa y el promedio global.

Particularmente, el caso de México deja ver que el trabajo (3.81) es importante solamente después del ámbito familiar (3.97). Además, es el segundo país que más considera a la actividad como valiosa, sólo por debajo de Ecuador (3.83). Incluso la media de valoración se localiza muy arriba del promedio americano (3.57), europeo (3.32) y mundial (3.47).

Para analizar con más detalle el comportamiento de la centralidad del trabajo relativa de México, pero de forma transversal, los autores del presente toman datos de otros países y continentes únicamente para hacer ejercicios referenciales que coadyuven a posicionar su caso de estudio para una revisión comparativa. Por ejemplo, en la com-

Tabla 1

Importancia relativa del trabajo según la Encuesta Mundial de Valores periodo 2010-2014. Medias aritméticas globales, América, México y Europa

\begin{tabular}{ccccccc}
\hline País & Trabajo & Familia & Amigos & Ocio & Política & Religión \\
\hline Ecuador & 3.83 & 3.98 & 3.03 & 3.41 & 2.45 & 3.52 \\
\hline México & 3.81 & 3.97 & 3.12 & 3.42 & 2.39 & 3.37 \\
\hline Colombia & 3.74 & 3.84 & 2.99 & 3.34 & 1.98 & 3.41 \\
\hline Perú & 3.66 & 3.84 & 2.77 & 3.09 & 2.74 & 3.29 \\
\hline Brasil & 3.61 & 3.87 & 3.14 & 3.19 & 2.25 & 3.40 \\
\hline Trinidad y Tobago & 3.58 & 3.93 & 3.08 & 3.35 & 2.23 & 3.69 \\
\hline Uruguay & 3.50 & 3.87 & 3.30 & 3.36 & 2.03 & 2.28 \\
\hline Chile & 3.46 & 3.90 & 3.13 & 3.46 & 1.94 & 2.70 \\
\hline Argentina & 3.42 & 3.88 & 3.43 & 3.13 & 2.12 & 2.55 \\
\hline Estados Unidos & 3.05 & 3.90 & 3.50 & 3.31 & 2.57 & 2.98 \\
\hline Media continental América & 3.57 & 3.90 & 3.15 & 3.31 & 2.23 & 3.12 \\
\hline Media continental Europa & 3.32 & 3.88 & 3.38 & 3.24 & 2.22 & 2.56 \\
\hline Media Mundial & 3.47 & 3.91 & 3.33 & 3.13 & 2.38 & 3.09 \\
\hline
\end{tabular}

Nota: -V8. Importancia del trabajo en la vida. 1. Nada importante; 2. Poco importante; 3. Bastante importante; 4. Muy importante.

Elaboración propia con datos de WVS (s. f.). 
Tabla 2

Posición jerárquica otorgada al trabajo frente a otros ámbitos de la vida (familia, amigos, ocio, política y religión) de la Encuesta Mundial de Valores en el periodo 1990 a 2014

\begin{tabular}{cccccc}
\hline País & $1990-1994$ & $1995-1999$ & 20002004 & $2005-2009$ & $2010-2014$ \\
\hline México & 2 & 2 & 2 & 2 & 2 \\
\hline Promedio América & 2 & 2 & 2 & 2 & 2 \\
\hline Promedio Europa & 2 & 2 & 3 & 3 & 3 \\
\hline Promedio mundial & 2 & 2 & 2 & 2 & 2 \\
\hline
\end{tabular}

Nota: $2=$ Segunda prioridad; $3=$ Tercera prioridad; $4=$ Cuarta prioridad.

Elaboración propia con datos de WVS (s. f.).

paración de submuestras por continente se observa que el valor trabajo en América señala una constante al ubicarse como segunda prioridad, siempre por debajo de la familia. El caso europeo se mantuvo en el mismo nivel hasta el año 2000, cuando pasó a tercera prioridad, por debajo de la familia y las amistades, con ello hay un desfase importante en cuanto a las tendencias globales, mientras que México se ha mantenido constante en la ubicación como segunda prioridad (tabla 2). Se observa que México mantiene una constante de valoración al trabajo ajustado a los niveles globales, americanos y arriba de los europeos en las últimas tres encuestas.

\section{Centralidad del trabajo y variables macroeconómicas} Una de las explicaciones de esta valoración diferenciada entre países la otorga la perspectiva teórica denominada Índice de Postmaterialidad, propuesta por Ronald Inglehart en los estudios realizados entre 1973 y 1990 . En ellos midió las prioridades valorativas de 43 sociedades con el fin de diagnosticar las preponderancias materialistas - posmaterialistas como polos culturales de las sociedades contemporáneas. Dicha teoría supone que sociedades altamente industrializadas, que han alcanzado a satisfacer las necesidades materiales inmediatas, priorizan como valores principales a la democracia, la libertad de expresión, la calidad de vida, el respeto a los derechos humanos, mayor participación política, en la que el dinero y la satisfacción de necesidades básicas, por ejemplo, no resultan aspectos fundamentales de su organización o demanda pública, a las que denomina culturas posmaterialistas, mientras que las de niveles inferiores de industrialización y que aún no alcanzan a proveer de las condiciones suficientes de subsistencia a sus ciudadanos le otorgan prioridad a valores referentes a la vida material inmediata como el trabajo, la búsqueda de seguridad personal y colectiva (como la lucha contra la delincuencia), mantenimiento estable de la economía, etcétera (Inglehart, 1991, 1998; Inglehart \& Abramson, 1994; Inglehart, \& Baker, 2000; Ros García, 2002).

Así, la relación entre desarrollo económico y las prioridades valorativas hace que la predicción a partir de cálculos estadísticos sea situada geográficamente. Por tanto, países y regiones altamente desarrollados se ubican en el rango posmaterialista, mientras que las no tan desarrolladas ni con indicadores macroeconómicos favorables son consideradas prioritariamente materialistas. En el caso de la centralidad del trabajo, valor considerado por otros estudios (Veira Veira, \& Muñoz Goy, 2004; Veira Veira, \& Romay Martínez, 1998) como indicador también de sociedades materialistas o posmaterialistas, resulta clave para ubicar a los países en cualquiera de los extremos, al formar conjuntos de naciones a partir de su coincidencia valorativa. Las diferencias en la valoración del trabajo como menos importante entre sociedades altamente industrializadas y con un estatus de referentes económicos en sus respectivos continentes permite ver la concordancia teórica con los resultados de la encuesta de valores.

Se puede observar en la figura 2 la correlación negativa $(R=0.76433)$ entre valor trabajo y Producto Interno Bruto per cápita, lo que hace congruentes los diagnósticos de la posmaterialidad. Por tanto, en los países de mayor distribución de la riqueza y desarrollo económico se tiende a valorar en menor medida al trabajo. Por tanto, una primera mirada deja ver que los países latinoamericanos valoran más el trabajo que los europeos. Particularmente se ve que Suecia, Holanda, Estados Unidos y Alemania son países con altos niveles de PIB per cápita promedio entre 2010-2014 y, a su vez, son los que en menor medida valoran al trabajo como muy importante. 


\section{IIVESTIGAGIÓn Y CUERGIA DE LA UחIVERSIDAD AUTÓNOMA DE RGUASCALIERTES}

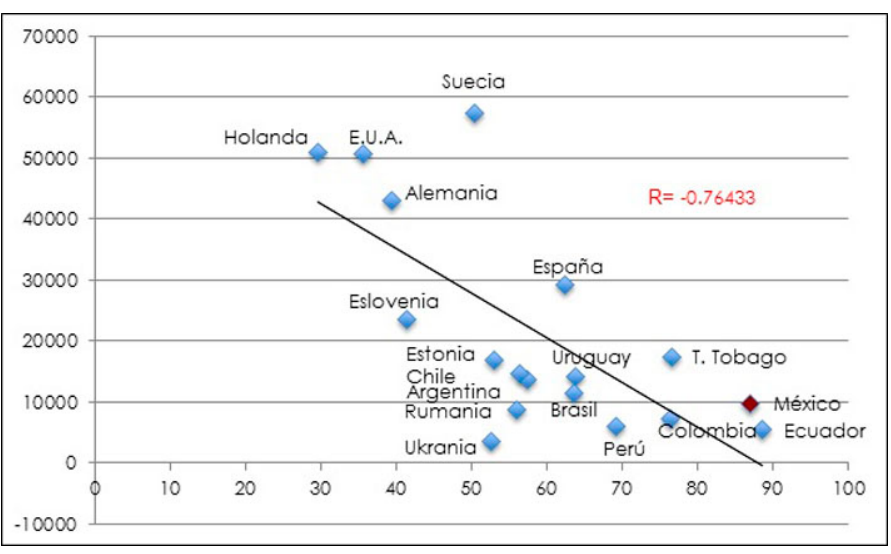

Figura 2. Dispersión. PIB per cápita y alta importancia al trabajo. Elaboración propia con datos de BM (s. f. b.) y WVS (s. f.).

En otro lado de la recta se ve a Ecuador, México y Colombia que valoran de forma significativa al trabajo pero posicionados en los bajos índices promedio de PIB per cápita en el mismo periodo. La segregación por submuestra europea responde a los mismos supuestos, ya que los países con bajos índices de PIB valoran en mayor medida al trabajo que los que se encuentran en condiciones contrarias. Por ejemplo Suecia, Alemania y Holanda en un extremo; en el otro Rumania, Estonia y Ucrania.

Con respeto al Índice de desigualdad GINI (Indicador de distribución del ingreso que fluctúa entre el valor 0 , que indica igualdad absoluta y 1 , desigualdad absoluta) los resultados no son tan distintos al ejercicio anterior, ya que el coeficiente de correlación señala 0.6539. En la figura 3 se aprecia la directriz. Por tanto, se ve que los países con mayor

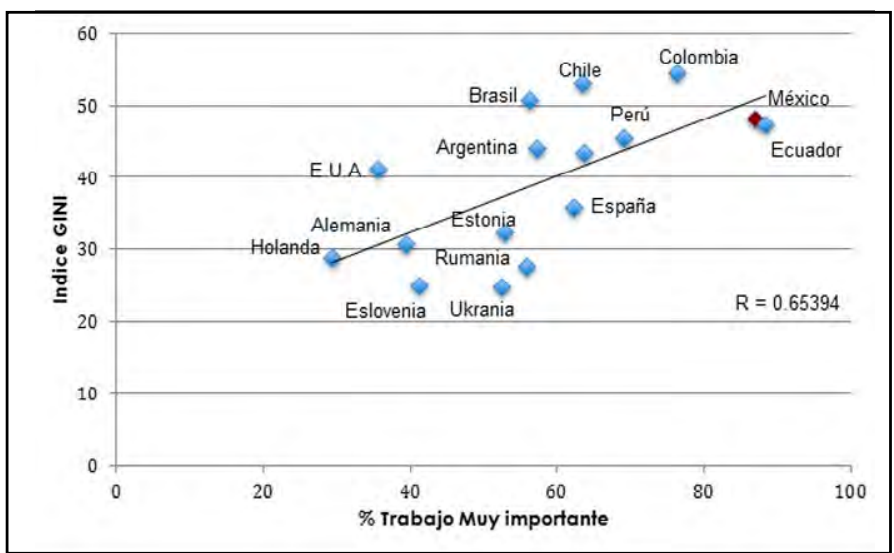

Figura 3. Dispersión. Índice GINI y alta importancia al trabajo. Elaboración propia con datos de BM (s. f. a) y WVS (s. f.). desigualdad como Ecuador, México, Colombia y Perú son los que valoran en mayor medida al trabajo como muy importante.

Otra variable macrosocial considerada para el análisis de correlación con el objetivo de ofrecer diagnósticos referentes a la teoría de la posmaterialidad (Ros García, 2002) es el índice de Desarrollo Humano (IDH), el cual testifica el desarrollo de un país a partir de los niveles de vida por cada ciudadano a partir de tres indicadores: 1) Esperanza de vida y tasa de mortalidad de menores; 2) escolaridad entendida a partir del nivel de alfabetización, así como porcentaje de personas con educación básica y 3) ingresos en PIB per cápita. El rango de indicador va desde el 35 hasta el 96 . El primer valor es el más bajo o con menos desarrollo humano y el segundo el más alto.

En la figura 4 se aprecia que la correlación es similar a la de anteriores revisiones, ya que la orientación es negativa, con valor de -0.7657759 . Por tanto, la relación indica que cuanto mayor IDH menor valoración al trabajo. Al mismo tiempo, la ubicación en la dispersión de los países con menores índices de desarrollo humano se agrupan en un sector de mayor centralidad al trabajo. Aquí se ubican Ecuador, México, Perú, Colombia y Trinidad y Tobago. México se ubica al extremo inferior de la línea de tendencia con países de bajo desarrollo humano y alta centralidad al trabajo; mientras que en el otro extremo de la recta se ubican Alemania, Holanda y Estados Unidos, lo que revela una correspondencia entre alto desarrollo humano y menor centralidad al trabajo.

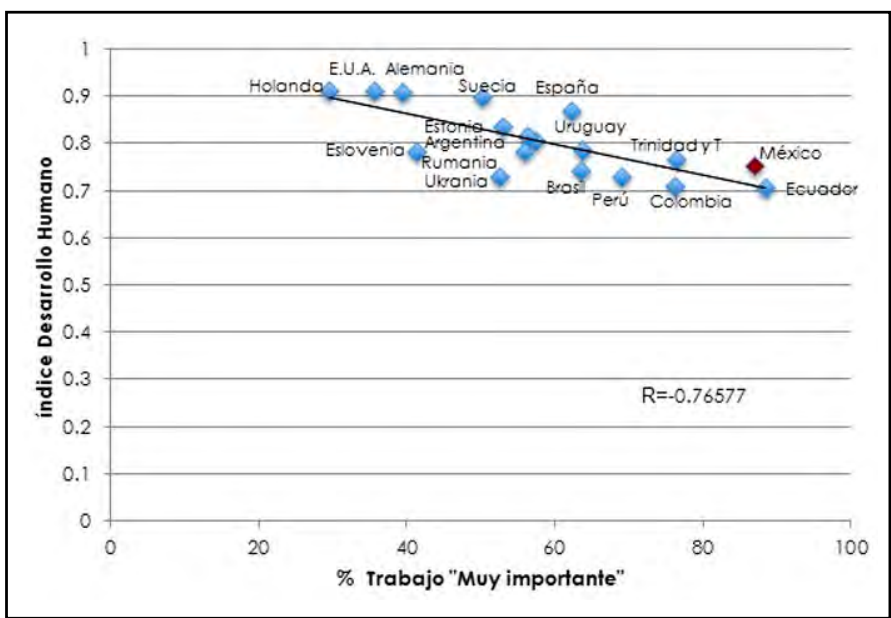

Figura 4. Índice de Desarrollo Humano y alta importancia al trabajo. Elaboración propia con datos de UN (s. f.) y WVS (s. f.). 
Tabla 3

Índice de posmaterialidad en México en porcentajes del periodo 1990-2014

\begin{tabular}{lccccc}
\hline & $1990-1994$ & $1995-1999$ & $2000-2004$ & $2005-2009$ & $2010-2014$ \\
\hline Materialismo & 50.0 & 19.0 & 25.0 & 19.0 & 17.0 \\
\hline Mixto & 35.0 & 59.0 & 54.0 & 59.0 & 62.0 \\
\hline Posmaterialismo & 2.0 & 16.0 & 12.0 & 19.0 & 20.0 \\
\hline
\end{tabular}

Nota: Elaboración propia con datos de WVS (s. f.).

\section{DISCUSION}

La importancia del trabajo en México tiende hacia un incremento exponencial, ha pasado de $66.9 \%$ a 87\%; lo cual admite las tesis de que el incremento de la importancia del trabajo es una tendencia clara y sostenida (De la Garza Toledo, 2010), a diferencia de lo que se diagnostica en países Europeos (Bauman, 2000, 2004; Beck, 2007). Sin embargo, las razones de tal proceso responden a la propuesta teórica de la posmaterialidad que supone: entre mayores niveles de bienestar material tenga una nación o país, su valoración se centrará en aspectos no materiales. El caso de México coincide en que sus condiciones macroeconómicas escasas influyen positivamente en la propensión a su alta valoración al trabajo como importante. Por tanto, pareciera que la sociedad mexicana se ubica en el grupo de culturas materialistas, tal como se ha vaticinado previamente (Inglehart, 1998). Esto, sin embargo, contrasta con los resultados que la propia lógica de razonamiento de la Encuesta Mundial de Valores ofrece mediante su índice que señala la propensión hacia la posmaterialidad. Empero, el carácter mixto es predominante en la mayoría de periodos de análisis (tabla 3).

A nivel general, el índice reporta el carácter mixto; a nivel específico, en cuanto al trabajo, se afirma su tendencia materialista. Tal paradoja se explica con base en los postulados de la propuesta teórica del posmaterialismo, ya que los agentes sociales determinan el mayor valor subjetivo a elementos de vida de los que más se carecen $y$, a medida en que sus condiciones económicas y sociales mejoran, la prioridad valorativa cambia. La hipótesis de la escasez avala que las prioridades valorativas de los agentes son el reflejo de las condiciones socioeconómicas de su entorno (Inglehart, 1991).

El ajuste entre mejores condiciones materiales con los valores posmaterialistas no es inmediato, ya que se requiere que subjetivamente se asuman como seguras las condiciones de vida para estimar a otros valores como prioritarios. Incluso resulta natural que en este proceso, en sociedades en las que prevalecen valores mixtos y posmaterialistas, se supongan los concernientes a seguridades materiales, como el trabajo, mientras los estándares de seguridad se extienden y asimilan en la población general (Díez Nicolás, 1994).

Otro hallazgo a considerar para debate es la tesis que coloca a los países altamente industrializados y con elevados niveles de desarrollo social como ejemplares en la alta estima al trabajo, a causa de la estrecha relación entre industrialización y alta importancia a la labor (Schwartz, 1999; Snir, \& Harpaz, 2005). Sin embargo, como se ha revisado, los resultados de esta exploración suponen que los países con niveles de desarrollo económico y social ínfimos son los que así lo señalan prioritariamente. Existen algunos antecedentes de investigaciones empíricas en contextos europeos que de igual manera asumen esta hipótesis, lo que demuestra la relación de bajos niveles macroeconómicos (figura 5) con alta valoración al trabajo (Veira Veira, \& Muñoz Goy, 2004; Veira Veira, \& Romay Martínez, 1998).

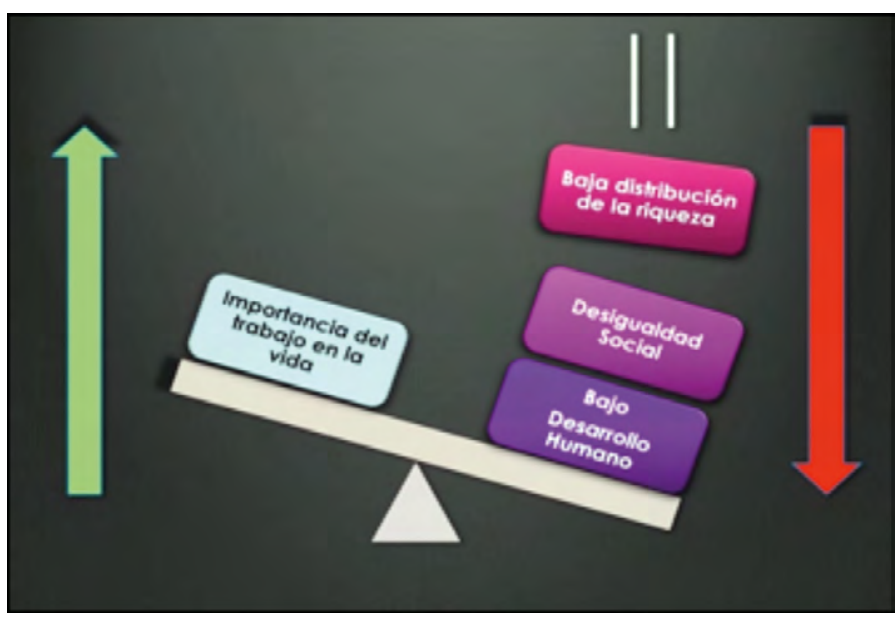

Figura 5. Correlación entre bajos niveles materiales y de vida con la importancia del trabajo en la vida.

Elaboración propia. 


\section{IIVESTIGAGIÓn Y CUERCIA DE LA UחIVERSIDAD AUTÓNOMA DE RGUASCHLIETTES}

\section{CONCLUSIONES}

La centralidad del trabajo en México ha sido verificada históricamente a partir de una encuesta que posibilita su medición, así como tendencias. Con ello, se encontró que hay un crecimiento exponencial de la importancia de la actividad en este país, al grado de ubicarse como la segunda prioridad, así como de posicionarse comparativamente entre los países que en mayor medida lo considera. Sin embargo, esto sólo es consecuencia de las ínfimas condiciones de desarrollo humano y material en las que se encuentra, ya que como se revisó empíricamente, existen correlaciones positivas que señalan que entre menor distribución de la riqueza, igualdad y desarrollo humano, la importancia del trabajo es mayor. El semblante inverso se da en países cuyos indicadores de distribución material e igualdad social son favorables.

\section{REFERENCIAS}

- Antunes, R. (2005). Los sentidos del trabajo. Ensayo sobre la afirmación y la negación del trabajo. Buenos Aires, Argentina: Ediciones Herramienta y Taller de Estudios Laborales.

- Arendt, H. (2014). La condición humana. Barcelona, España: Paidós.

- Arriaza Balmón, M. (2006). Guía práctica de análisis de datos. Córdoba, España: Junta de Andalucía.

- Banco Mundial (BM). (s. f. a). Índice GINI 2010-2014. Recuperado el 16 de abril de 2016, de http://datos. bancomundial.org/indicador/SI.POV.GINI

-

(s. f. b). PIB per cápita 2010-2014. Recuperado el 16 de abril de 2016, de http://datos.bancomundial.org/ indicador/ny.gdp.pcap.cd

- Bauman, Z. (2000). Trabajo, consumismo y nuevos pobres. Barcelona, España: Gedisa Editorial.

- $\quad$ (2004). Modernidad líquida. Buenos Aires, Argentina: Fondo de Cultura Económica.

- Beck, U. (2007). Un nuevo mundo feliz. La precariedad del trabajo en la era de la globalización. Barcelona, España: Paidós.

- Davoine, L., \& Méda, D. (2008). Place et sens du travail en Europe: une singularité française? Document de Travail No. 96-1. France: Centre D'études de l'emploi.

- De la Garza Toledo, E. (2010). Hacia un concepto ampliado de trabajo. Del concepto clásico al no clásico. Barcelona, España: Anthropos-Universidad Autónoma Metropolitana Iztapalapa.

- Díez Nicolás, J. (1994). Post-materialismo y desarrollo económico en España. En J. Diez \& R. Inglehart (Coords.), Tendencias mundiales de cambio en los valores sociales y políticos. Madrid, España: FUNDESCO.
- García Martínez, J. M. A., \& Berrios Martos, M. P. (1999). E significado del trabajo en personas con patrón de conducta tipo A. Revista Psicothema, 11 (2), 357-366.

- Gorz, A. (1995). Metamorfosis del trabajo. Madrid, España: Editorial Sistema.

- Guijarro Martínez, F. (2013). Estadísitica aplicada a la valoración. Modelos multivariantes. Valencia, España: Universitat Politécnica de Valencia.

- Habermas, J. (1992). Teoría de la acción comunicativa II. Crítica de la razón funcionalista. Madrid, España: Taurus.

(1999). Teoría de la acción comunicativa I. Racionalidad de la acción y racionalización social. Madrid, España: Taurus. Península. (2002). Ensayos políticos. Barcelona, España:

- Inglehart, R. (1971). The silent revolution in Europe: Integenerational change in post-industrial societies. American Political Science Review, 65(4), 991-1017.

$\bullet$ industriales avanzadas. Madrid, España: Centro de Investigaciones Sociológicas. (1998). Modernización y posmodernización. El cambio cultural, económico y político en 43 sociedades. Madrid, España: Centro de Investigaciones Sociológicas-Siglo XXI editores. change. American Political Science Review, 88(2), 336-354.

- Inglehart, R., \& Baker, W. E. (2000). Modernization, cultural change, and the persistence of traditional values. American Sociological Review, 65(1), 19-51. 
- Kanungo, R. N. (1982). Measurement of job and work involvement. Journal of Applied Psychology, 67(3), 341-349.

- _ (1983). Work alienation. A pancultural perspective. International Studies of Management \& Organization, 13(1-2), 119-138.

- Köhler, H. D., \& Martín Artiles, A. (2010). Manual de la sociología del trabajo y de las relaciones laborales. Madrid, España: Publicaciones Delta.

- Marx, K. (1984). Manuscritos de economía y filosofía. Madrid, España: Alianza Editorial.

- Méda, D. (1998). El trabajo: un valor en peligro de extinción. Barcelona, España: Gedisa.

- \& Vendramin, P. (2013). Réinventer le travail. Paris, France: Preses Universitaires de France.

- Molina, J., \& Valenzuela, H. (2007). Invitación a la antropología económica. Barcelona, España: Ediciones Bellaterra.

- MOW International Research Team. (1987). The Meaning of Working. London, England: Academic Press.

- Noguera, J. A. (2000). El problema de la definición del trabajo. I Encuentros entre Humanidades y Ciencias Sociales. Recuperado el 25 de noviembre de 2013, de http://gsadi. uab.cat/images/pdfs/noguera/El\%20problema\%20de\%20 la\%20definici\%C3\%B3n\%20del\%20trabajo.pdf (2002). El concepto de trabajo y la teoría social crítica. Papers Revista de Sociología, 68, 141-168.

- Rieznik, P. (2007). Las formas de trabajo y la historia. Una introducción al estudio de la economía política. Buenos Aires, Argentina: Editorial Biblos.

- Ros García, M. (2002). Los valores culturales y el desarrollo socioeconómico: una comparación entre teorías culturales. Revista Española de Investigaciones Sociológicas, 99, 9-33.

- Sahlins, M. (1997). Cultura y razón práctica. Contra el utilitarismo en la teoría antropológica. Barcelona, España: Gedisa.
- Sanchis Gómez, E. (2004). Concepciones del trabajo: de las ambigüedades medievales a las paradojas actuales. Cuadernos de Relaciones Laborales, 22(1), 37-65.

- Schwartz, S. (1999). A theory of cultural values and some implications for work. Applied Psychology: An International Review, 48(1), 23-47.

- Smith, A. (2010). Investigación sobre la naturaleza y causa de la riqueza de las naciones. México: Fondo de Cultura Económica.

- Snir, R. (2011). To work or not to work: The measurement of Nonfinancial Employment Commitment. International Journal of Business and Social Science, 2(16), 271-278.

\& Harpaz, I. (2002). Work-leisure relations: leisure orientation and the meaning of work. Journal of Leisure Research, 34(2), 178-202. (2005). Test-retest reliability of the relative work centrality measure. Psychological Reports, 97, 569-572.

- United Nations (UN). (s. f.). United Nations Development Programe. Human Development Reports 2010-2014. Recuperado el 16 de abril de 2016, de http://hdr.undp.org/es

- Veblen, T. (2005). Teoría de la clase ociosa. México: Fondo de Cultura Económica.

- Veira Veira, J. L., \& Muñoz Goy, C. (2004). Valores y actitudes del trabajo en Europa Occidental. Revista Española de Sociología, 4, 51-66.

- Veira Veira, J. L., \& Romay Martínez, J. (1998). La cultura del trabajo y el cambio de valores. Sociológica: Revista de pensamiento social, 3, 23-38.

- Weber, M. (1999). La ética protestante y el espíritu del capitalismo. México: Colofón, S. A.

- WVS (World Values Survey Association). (s. f.). World Values Survey Wave 6, 2010-2014. Official Aggregate v.20150418. Recuperado el 5 de abril de 2014, de http://www. worldvaluessurvey.org/WVSOnline.jsp 